\title{
THE DIGITAL IMAGE CORRELATION METHOD APPLIED TO STUDYING THE LO- CALIZATION OF THE PLASTIC DEFORMATION OF AN ALLUMINUM ALLOY ELECTROLYTICALLY SATURATED WITH HYDROGEN
}

\author{
A. V. Bochkareva ${ }^{1,2}$, A. G. Lunev ${ }^{1,2}$, Yu. V. Li ${ }^{1}$, S. A. Barannikova ${ }^{1,3 *}$, L. B. Zuev ${ }^{1,3}$ \\ ${ }^{1}$ Institute of Strength Physics and Materials Science, 2/4 Akademicheskii ave., 634055, Tomsk, Russian Federation \\ ${ }^{3}$ Tomsk Polytechnic University, 30 Lenina ave., 634050, Tomsk, Russian Federation \\ ${ }^{2}$ Tomsk State University, 36 Lenina ave., 634050, Tomsk, Russian Federation
}

*Corresponding author. E-mail: bsa@ispms.tsc.ru; address for correspondence: 2/4, pr. Akademicheskii, 634055 , Tomsk, Russian Federation. Tel.: (3822)286923

The paper presents investigations of the effect of hydrogen embrittlement on the plastic flow. The studies are performed for test samples of aluminum alloy subjected to electrolytic hydrogenation in a three-electrode electrochemical cell at a controlled constant cathode potential using the Digital Image Correlation method. Diagrams of localized plasticity wave propagation through the length of the sample under deformation have been obtained. The deformation diagrams are examined for the deformed samples of the aluminum alloy and also main parameters of localized plasticity patterns are determined. Using the scanning electron microscopy method, the changes in the fracture surface are investigated.

Keywords: hydrogen embrittlement, duralumin, plasticity, microhardness, localized deformation.

DOI: $10.17804 / 2410-9908.2016 .1 .045-054$

\section{References}

1. Semenova I.V., Florianovich G.M., Khoroshilov A.V. Korroziya i zashchita ot korrozii. [Corrosion and Corrosion Prevention]. Moskow, Fizmatlit Publ., 2002, 335. (In Russian).

2. Lunarska E., Chernyaeva O. Effect of precipitates on hydrogen transport and hydrogen embrittlement of aluminum alloys. Materials Science, 2004, vol. 40, iss. 3, pp. 399-407. DOI: $10.1007 / \mathrm{s} 11003-005-0049-2$.

3. Kannan M., Raja V.S. Hydrogen embrittlement susceptibility of over aged 7010 Al-alloy. Journal of Materials Science, 2006, vol. 41, pp. 5495-5499. DOI: 10.1007/s10853-006-0287-1.

4. Kim S.J., Han M.S., Jang S.K. Electrochemical characteristics of Al-Mg alloy in seawater for leisure ship: Stress corrosion cracking and hydrogen embrittlement. Korean Journal of Chemical Engineering, 2009, vol. 26, iss. 1, pp. 250-257. DOI: 10.1007/s11814-009-0042-9.

5. Kumar S., Namboodhiri T.K.G. Precipitation hardening and hydrogen embrittlement of aluminum alloy AA7020. Bulletin of Materials Science, 2011, vol. 34, no. 2, pp. 311-321. DOI: $10.1007 / \mathrm{s} 12034-011-0066-8$.

6. Nykyforchyn H.M., Ostash O.P., Tsyrul'nyk O.T., Andreiko I.M., Holovatyuk Yu.V. Electrochemical evaluation of the in-service degradation of an aircraft aluminum alloy. Materials Science, 2008, vol. 44, iss. 2, pp. 254-259. DOI: 10.1007/s11003-008-9067-1.

7. Plekhov O.A., Naimark O., Saintier N. Experimental study of energy accumulation and dissipation in iron in an elastic-plastic transition. Technical Physics, 2007, vol. 52, no. 9, pp. 1236-1238. DOI: 10.1134/S106378420709023X.

8. Tretyakova T.V., Vildeman V.E. Studying Cracks Development under Complex Loading Conditions by Digital Image Correlation. Zavodskaya Laboratoriya. Diagnostika Materialov, 2012, vol. 78, no. 6, pp. 54-58. (In Russian). 
9. Shibkov A.A., Zolotov A.E., Zheltov M.A. Mechanisms of the nucleation of macrolocalized deformation bands. Izvestiya RAN. Seriya Fizicheskaya, 2012, vol. 76, no.1, pp. 85-95. (In Russian).

10. Danilov V.I., Bochkaryova A.V., Zuev L.B. Macrolocalization of deformation in material having unstable plastic flow behavior. The Physics of Metals and Metallography, 2009, vol. 107, iss. 6, pp. 616-623. DOI: 10.1134/S0031918X0906012X.

11. Zuev L.B., Gorbatenko V.V., Pavlichev K.V. Elaboration of speckle photography techniques for plastic flow analyses. Measurement Science and Technology, 2010, vol. 21, no. 5, pp. 1-5. DOI: 10.1088/0957-0233/21/5/054014.

12. Takai K., Shoda H., Suzuki H., Nagumo M. Lattice defects dominating hydrogen-related failure of metals. Acta Materialia, 2008, vol. 56, iss. 18, pp. 5158-5167. DOI: 10.1016/j.actamat.2008.06.031.

13. Yagodzinskyy Y., Todoshchenko O., Papula S., Hänninen H. Hydrogen Solubility and Diffusion in Austenitic Stainless Steels Studied with Thermal Desorption Spectroscopy. Steel Research International, 2011, vol. 82, iss. 1, pp. 20-25. DOI: 10.1002/srin.201000227.

14. Barannikova S.A., Nadezhkin M.V., Mel'nichuk V.A., Zuev L.B. Tensile plastic strain localization in single crystals of austenite steel electrolytically saturated with hydrogen. Technical Physics Letters, 2011, vol. 37, no. 9, pp. 793-796. DOI: 10.1134/S1063785011090057.

15. Barannikova S.A., Nadezhkin M.V., Lunev A.G., Gorbatenko V.V., Shlyakhova G.V., Zuev L.B. Effect of hydrogen on the localization of plastic deformation under tensile of low-carbon steel. Metallofizica i Noveyshie Tekhnologii, 2014, vol. 36, iss. 2, pp. 229-245. (In Russian).

16. Barannikova S.A., Nadezhkin M.V., Lunev A.G., Gorbatenko V. V., Zuev L.B. Regularities in localization of plastic flow upon electrolytic hydrogenation of an iron bcc-alloy. Technical Physics Letters, 2014, vol. 40, iss. 3, pp. 211-214. DOI: 10.1134/S1063785014030043.

17. Birnbaum H.K., Sofronis P. Hydrogen-enhanced localized plasticity - a mechanism for hydrogen-related fracture. Material Science and Engineering: A, 1994, vol. 176, iss. 1-2, pp. 191-202. DOI: 10.1016/0921-5093(94)90975-X.

18. McDonald R.J., Efstathiou C., Curath P. The wave-like plastic deformation of single crystals copper. Journal of Engineering Materials and Technology, 2009, vol. 131, iss. 3, pp. 692-703. DOI: $10.1115 / 1.3120410$.

19. Asharia A., Beaudoin A., Miller R. New perspectives in plasticity theory: dislocation nucleation, waves and partial continuity of plastic strain rate. Mathematics and Mechanics of Solids, 2008, vol. 13, no. 3-4, pp. 292-315. DOI: 10.1177/1081286507086903.

20. Fressengeas C., Beaudoin A., Entemeyer D. Lebedkina T., Lebyodkin M., Taupin V. Dislocation transport and intermittency in the plasticity of crystalline solids. Physical Review B, 2009, vol. 79, iss.1, pp. 014108-1-014108-9. DOI: 10.1103/PhysRevB.79.014108. 
Подана в журнал: 25.12 .2015

УДК 621.778:539.37

DOI: $10.17804 / 2410-9908.2016 .1 .045-054$

\title{
ИССЛЕДОВАНИЕ ЛОКАЛИЗАЦИИ ПЛАСТИЧЕСКОЙ ДЕФОРМАЦИИ ЭЛЕКТРОЛИТИЧЕСКИ НАСЫЩЕННОГО ВОДОРОДОМ АЛЮМИНИЕВОГО СПЛАВА МЕТОДОМ ДЕКОРРЕЛЯЦИИ ЦИФРОВЫХ СПЕКЛ-ИЗОБРАЖЕНИЙ
}

\author{
А. В. Бочкарёва ${ }^{1,2}$, А. Г. Лунев ${ }^{1,2}$, Ю. В. Ли ${ }^{1}$, С. А. Баранникова ${ }^{1,3}$, Л. Б. Зуев ${ }^{1,3}$ \\ ${ }^{1}$ Институт физики прочности и материаловедения СО РАН, пр. Академический, 2/4, Томск, \\ Российская Федераиия \\ ${ }^{2}$ НИ Томский политехнический университет, пр. Ленина, 30, г. Томск, Российская Федерация \\ ${ }^{3}$ НИ Томский государственный университет, пр. Ленина, 36, г. Томск, Российская Федерация
}

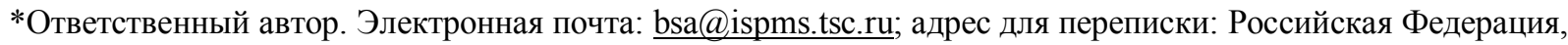
г. Томск, пр. Академический, 2/4, 634055. Телефон: (3822) 28-69-23

В работе исследовано влияние насыщения водородом алюминиевого сплава марки Д1 электролитическим методом на локализацию пластического течения. Методом декорреляции цифровых спекл-изображений получены эволюционные картины распространения фронтов локализации по длине образца во время механических испытаний. Проведен анализ стадийности кривых пластического течения и определены основные количественные параметры распространения локализованных зон. Приведены результаты фрактографических исследований поверхностей разрушения дюралюмина в исходном состоянии и после взаимодействия с агрессивной водородосодержащей средой.

Ключевые слова: водородное охрупчивание, дуралюмин, пластичность, микротвердость, локализачия деформачии .

\section{1. Введение}

Изучение влияния коррозионных процессов на механические характеристики и параметры локализации пластической деформации конструкционных металлов и сплавов представляет собой важную инженерную задачу [1]. Термически упрочняемые алюминиевые сплавы, к которым, безусловно, относят дуралюмины, обладают достаточными прочностными характеристиками и одновременно высокой степенью сопротивления коррозионным процессам, ввиду наличия на поверхности инертной оксидной пленки. Все вышесказанное, а также относительная доступность алюминия и его сплавов, позволяют находить широкое применение данным материалам во многих отраслях промышленности, в том числе авиационной, кораблестроительной, в качестве конструкционных узлов различного рода сооружений и др. Широкое применение алюминиевых сплавов предполагает возможность воздействия на них различных факторов окружающей среды, которые инициируют утонение верхнего слоя защитной оксидной пленки, что приводит к развитию коррозионных процессов и снижает общую безопасность конструкций. Алюминиевые сплавы в целом инертны к коррозионным процессам, однако это справедливо только лишь для сплавов с малой степенью пересыщения твердого раствора. Сплавы с высокой концентрацией легирующих элементов, к которым относят сплавы системы дуралюмин, при условии существования в агрессивных средах, напротив, подвержены коррозионному растрескиванию. Причиной такого растрескивания может служить в том числе водород [2-6]. В связи с этим представляется важным установить влияние насыщения водородом электролитическим методом на деформационное поведение термически упрочняемых алюминиевых сплавов.

Применение новых методов для анализа неоднородности полей деформаций позволило значительно продвинуться в понимании природы локализации пластической деформации Bochkareva A.V. et al. / The digital image correlation method applied to studying the localization of the plastic deformation of an alluminum alloy electrolytically saturated with hydrogen. 
и разрушения металлов и сплавов $[7,8]$. Несмотря на многочисленные исследования процесса макролокализации деформации при прерывистой текучести в виде образования деформационных полос на поверхности нагружаемых образцов в алюминиевых сплавах [9] к настоящему моменту времени многие вопросы остаются открытыми. Экспериментальные результаты [10], на которых базируется автоволновой подход к описанию локализованной пластической деформации, получены с использованием уникального измерительного комплекса ALMEC для фотографической регистрации спекл-изображения [11].

B настоящей работе применение комплекса ALMEC-tv для цифровой регистрации спекл-изображения позволило повысить быстродействие прибора, обеспечить возможность регистрации процессов формоизменения и разрушения в реальном времени, а также повысить пространственное разрешение при исследовании неоднородности фронтов деформации в алюминиевом сплаве Д1 при одноосном растяжении в исходном состоянии и подверженного электролитическому насыщению водородом в течение 100 ч. [12]. Цель работы заключалась в исследовании влияния насыщения водородом алюминиевого сплава марки Д1 электролитическим методом на локализацию пластического течения и изменение механических свойств, поскольку наводороживание алюминиевых сплавов инициирует различные структурные изменения.

\section{2. Материалы и методика эксперимента}

Для исследований выбран дисперсионно-твердеющий сплав системы дуралюмин Д1. После штампования из горячекатаного листа образцы были подвергнуты искусственному старению при $\mathrm{T}=610 \mathrm{~K}$ в течение 3 ч с охлаждением в печи (состояние 1$)$.

Наводороживание образцов осуществляли электролитическим способом посредством помещения образцов в трехэлектродную электрохимическую ячейку при постоянном катодном потенциале $U=-600$ мB, задаваемом относительно хлорсеребряного электрода сравнения, в $1 \mathrm{~N}$ раствор серной кислоты с добавлением катализатора - тиомочевины в концентрации 20 мг/л при температуре Т=323 К в течение 100 часов [13] (состояние 2).

Механические испытания образцов в форме двойной лопатки с размерами рабочей части $50 \times 10 \times 2$ мм проводились при $300 \mathrm{~K}$ по схеме одноосного растяжения со скоростью растяжения $6,67 \times 10^{-5} \mathrm{c}^{-1}$ на испытательной машине LFM-125, снабженной для изучения макролокализации деформации автоматизированным лазерным измерительным комплексом ALMEC-tv (ИФПМ CO РАН, Россия) для анализа цифровых спекл-изображений .

Микротвердость сплава Д1 в исходном состоянии и после его насыщения атомами водорода измерялась стандартным методом с использованием наконечника Виккерса на микротвердомере ПМТ-3. Нагрузка на индентор составила $\mathrm{P}=0,15 \mathrm{H}$. Индентированию подвергалась полированная поверхность рабочей части образца. Полученные данные представлены с использованием стандартных приемов математической обработки результатов физического эксперимента.

Фактографическое исследование поверхностей излома проводилось с применением растрового электронного микроскопа LEO EVO 50 (Zeiss, Германия) в ЦКП “Нанотех" ИФПМ СО РАН.

\section{3. Результаты исследований и их обсуждение}

Кривые нагружения сплава Д1 в исходном состоянии (1) и подверженного электролитическому насыщению водородом в течение 100 ч (2), представленные на рис. 1, имеют пилообразный вид. Спады напряжений на диаграмме достигают 4-5 МПа, природа которых обусловлена эффектом Портевена - Ле Шателье. Без учета зубчатости подобные кривые можно отнести к диаграммам общего типа, которые принято описывать параболической функцией вида: 


$$
\sigma=\sigma_{0}+K \varepsilon^{n}
$$

где $K$ - коэффициент деформационного упрочнения; $n \leq 1-$ показатель деформационного упрочнения; $\sigma$ - условное напряжение, МПа; $\sigma_{0}$ - напряжение течения, МПа; $\varepsilon-$ условная деформация.

Использование метода логарифмирования, для реализации которого необходимо перевести условные напряжение и деформацию $(\sigma, \varepsilon)$ в истинные напряжение и деформацию $(s, e)$ чтобы исключить влияние геометрической составляющей пластической деформации плоского образца, позволяет преобразовать выражение (1) и представить кривую нагружения в системе логарифмических координат $\ln \left(s-s_{0}\right)=f(\ln e)$, где $s$ - истинное напряжение определяется как $s=\sigma(1+\varepsilon), \mathrm{s}_{0}-$ критическое напряжение сдвига, МПа; $e$ - истинная деформация, определяется как $e=\ln (1+\varepsilon)$. Прямолинейные участки на кривой нагружения в системе функциональных логарифмических координат, где показатель $n$ является постоянным и меняется дискретно от участка к участку, соответствуют стадиям пластического течения.

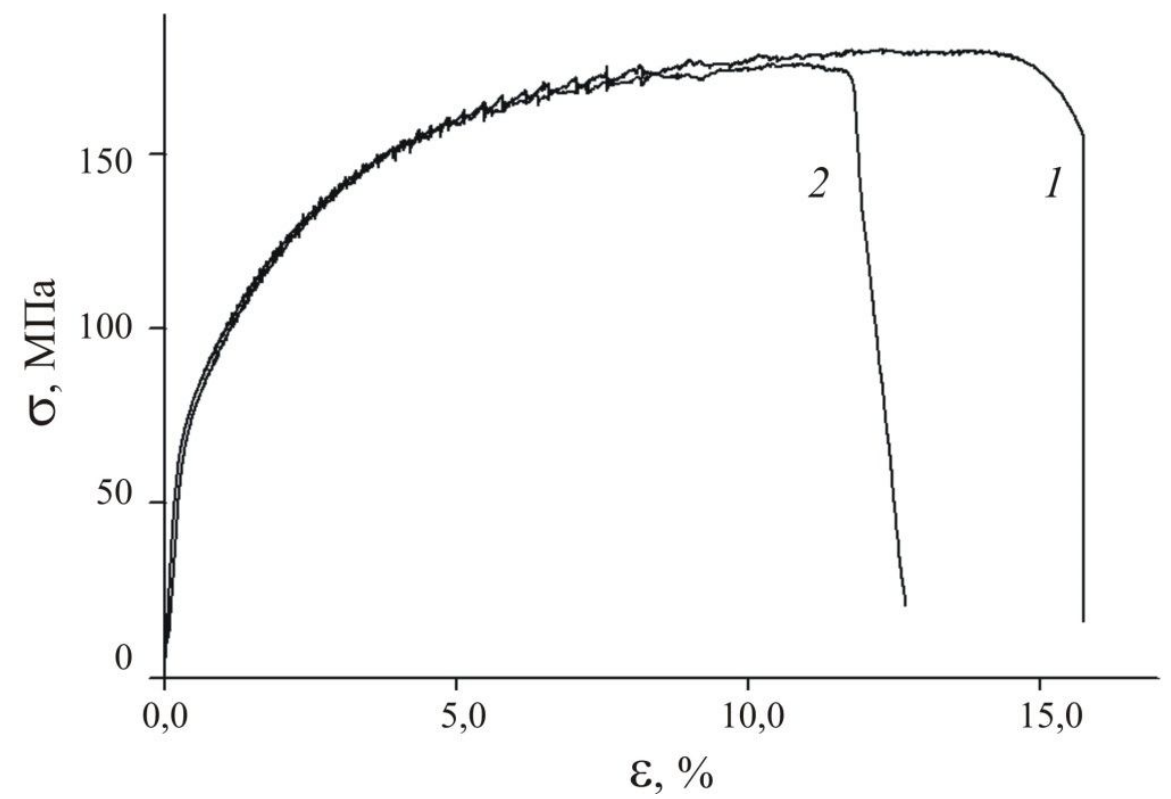

Рис.1. Кривые нагружения сплава Д1 в исходном состоянии (1) и после электролитического наводороживания $\mathrm{t}=100$ ч (2)

Коррозионные свойства полуфабрикатов из сплавов системы $\mathrm{Al}-\mathrm{Cu}-\mathrm{Mg}$ определяются фазовым составом выделений, их распределением по границе и объему зерна, а также степенью обеднения приграничных областей медью. Последнее обстоятельство в основном определяется условиями термической обработки.

Количественные данные механических характеристик: $\sigma_{0,2}-$ условный предел текучести, МПа; $\sigma_{B}$ - предел прочности, МПа; $\delta$ - относительное удлинение до разрыва, \% (таблица). Деформирование сплава Д1 в исходном состоянии (1) заканчивается образованием макроскопической шейки, свидетельствующей о приближении вязкого разрушения. Анализ стадийности деформационных кривых сплава в исходном состоянии в функциональных логарифмических координатах позволил достаточно четко выделить три продолжительных прямолинейных участка, для которых показатель деформационного упрочнения $n$ остается постоянным. Таким образом, на диаграмме нагружения сплава в исходном состоянии удалось выделить три стадии пластического течения: стадию линейного упрочнения с 
показателем $n=1$ на участке $\varepsilon=1,3 \div 2,3 \%$; стадию параболического упрочнения, где $n \approx 1 / 2$ на интервале $\varepsilon=3,6 \div 6,1 \%$ и стадию предразрушения с показателем $n \approx 0,3$ на участке $\varepsilon=6,9 \div 13,8 \%$. Полученные результаты согласуются с данными выполненных ранее исследований [10].

Механические характеристики сплава Д1 в исходном состоянии (1), после электролитического насыщения водородом в течение 100 ч (2)

\begin{tabular}{|c|c|c|c|c|c|c|c|c|c|c|}
\hline \multirow{2}{*}{ 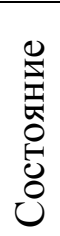 } & \multirow{2}{*}{$\begin{array}{l}\sigma_{0,2} \\
\mathrm{MPa}\end{array}$} & \multirow{2}{*}{$\begin{array}{l}\sigma_{\mathrm{B}} \\
\mathrm{MPa}\end{array}$} & \multirow[t]{2}{*}{$\delta, \%$} & \multirow{2}{*}{$\begin{array}{l}\mathrm{H} \mu \\
\mathrm{MПа}\end{array}$} & \multicolumn{2}{|c|}{$\begin{array}{c}\text { Линейная } \\
\text { стадия, } \\
\text { n } \approx 1\end{array}$} & \multicolumn{2}{|c|}{$\begin{array}{c}\text { Параболическая } \\
\text { стадия, } \mathrm{n} \approx 1 / 2\end{array}$} & \multicolumn{2}{|c|}{$\begin{array}{c}\text { Стадия } \\
\text { предразрушения, } \\
\mathrm{n} \leq 0,3\end{array}$} \\
\hline & & & & & $\varepsilon_{\mathrm{H}}, \%$ & $\varepsilon_{\kappa}, \%$ & $\varepsilon_{\mathrm{H}}, \%$ & $\varepsilon_{\mathrm{K}}, \%$ & $\varepsilon_{\mathrm{H}}, \%$ & $\varepsilon_{\mathrm{K}}, \%$ \\
\hline 1 & 76 & 180 & 15 & 251 & 1,3 & 2,3 & 3,6 & 6,1 & 6,9 & 13,8 \\
\hline 2 & 77 & 175 & 12 & 272 & 1,1 & 2,4 & 3,4 & 5,5 & 5,9 & 11,1 \\
\hline
\end{tabular}

Анализ кривой нагружения сплава после электролитического насыщения водородом в течение 100 ч показал наличие трех стадий деформационного упрочнения - линейной, параболической и стадии предразрушения. При этом сокращается их продолжительность, поскольку насыщение водородом привело к уменьшению пластичности (таблица).

Фрактографический анализ поверхностей изломов показал, что для образцов исследуемого сплава в исходном состоянии характерно формирование вытянутых ямок сдвига, тогда как поверхность разрушения образцов, подверженных электролитическому наводороживанию, представляет собой преимущественно систему равноосных ямок и ямок отрыва (рис. 2). Для исследуемых состояний 1 и 2 средний размер ямок отрыва $\bar{x}$ составил 7,6 $\pm 0,9$ мкм и $8,2 \pm 1,3$ мкм. Статистическая обработка данных методом двойного t-критерия показала, что полученные значения отличаются незначительно, однако анализ индивидуальных размеров ямок отрыва позволил установить, что при одинаковом минимальном размере сформированных пор $x \approx 1,5$ мкм максимальные размеры возрастают и составляют 15 и 19 мкм соответственно.

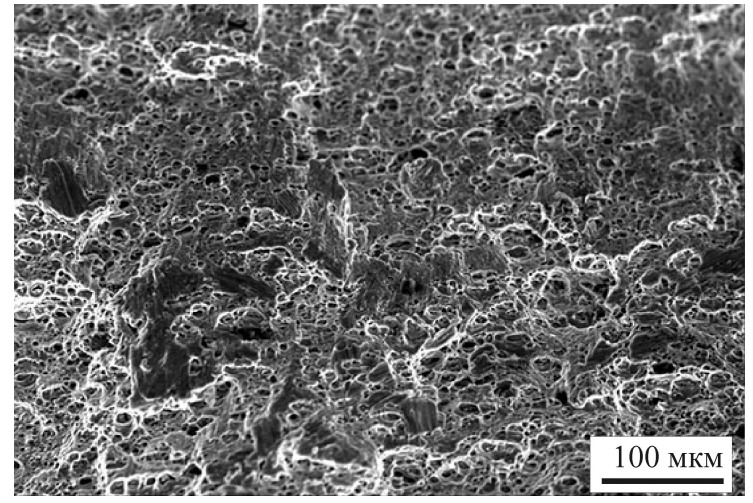

$a$

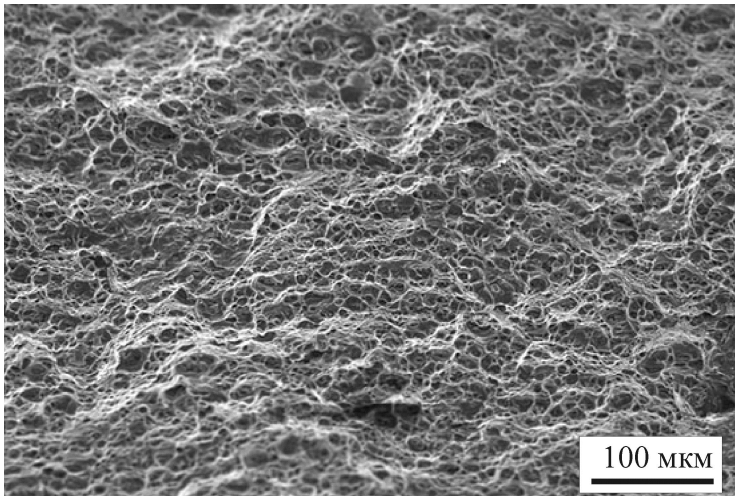

6

Рис. 2. Рельеф поверхности изломов Д1: $a-$ в состоянии (1); $\sigma$ - в состоянии (2)

В [14-16] показано, что пластическая деформация твердых тел развивается локализовано на всем протяжении процесса течения. Особенно эффектно она проявляется на макроскопическом масштабном уровне, когда картины локализации связаны с законом деформационного упрочнения $\theta(\varepsilon)$, действующим на соответствующей стадии процесса. В этом случае картины локализации принимают форму автоволн разных типов, причем существует вза- 
имно однозначное соответствие между типом картины локализации и законом деформационного упрочнения, действующим на этой стадии процесса течения.

При обработке цифровых спекл-изображений методикой, основанной на измерении скорости мерцания спеклов, наблюдаются периодически перемещающиеся одиночные фронты локализованной деформации, аналогичные полосам Чернова-Людерса. Пример таких фронтов локализации пластической деформации показан на рис. 3. Из рисунка хорошо видно, что используемый метод декорреляции цифровых спекл-изображений рабочей поверхности деформируемого сплава Д1 в самом начале участка пластического течения (состояние 1 , $\varepsilon=1,1 \%$ рис. $3 a$, а также после электролитического наводорживания исследуемых образцов при значительных пластических деформациях (состояние $2, \varepsilon=5,1 \%$ ) рис. 36 , позволяет одинаково четко визуализировать области локализации пластической деформации и определить местоположение очага на рабочей части.

Последовательность координат X, мм, соответствующих положению полос деформации на рабочей поверхности образца вдоль оси растяжения, с течением времени $\mathrm{t}$, с аппроксимировали прямыми линиями. Из зависимостей $\mathrm{X}(\mathrm{t})$ определяются скорость и расстояние между полосами локализованной деформации, которые служат информативным дополнением к обычным механическим характеристикам материала. Скорость распространения зон локализации пластической деформации составила для исходного состояния $\sim(0,2 \ldots 1,8) \cdot 10^{-3} \mathrm{M} / \mathrm{c}$ и для наводороженного состояния составила $(0,3 \ldots 2,0) \cdot 10^{-3} \mathrm{M} / \mathrm{c}$.

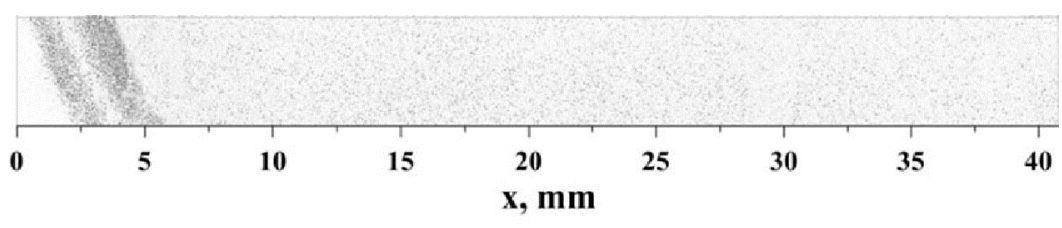

$a$

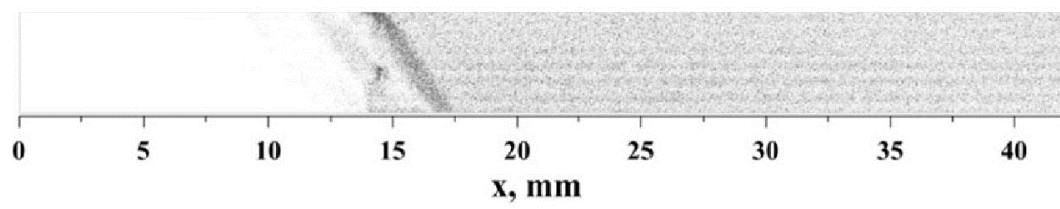

6

Рис. 3. Визуализация полос макролокализации деформации на поверхности образца Д1 в состоянии (1) при $\varepsilon=1,1 \%(a)$ и в состоянии (2) при $\varepsilon=5,1 \%$ (б)

Анализ картин локализации пластической деформации в образцах сплава Д1 показал преимущественное распространение одиночных фронтов деформации по всей длине образца. Зарождение новых полос локализованной пластической деформации происходит вблизи неподвижного захвата (рис. 4). Скорость полос локализации пластической деформации и расстояние между ними снижаются по мере роста общей деформации как в исходном состоянии (1), так и после предварительного насыщения водородом (2). На стадии предразрушения к моменту формирования шейки перемещение фронтов деформации ограничено узкой областью - местом будущего разрушения (рис. 4). 

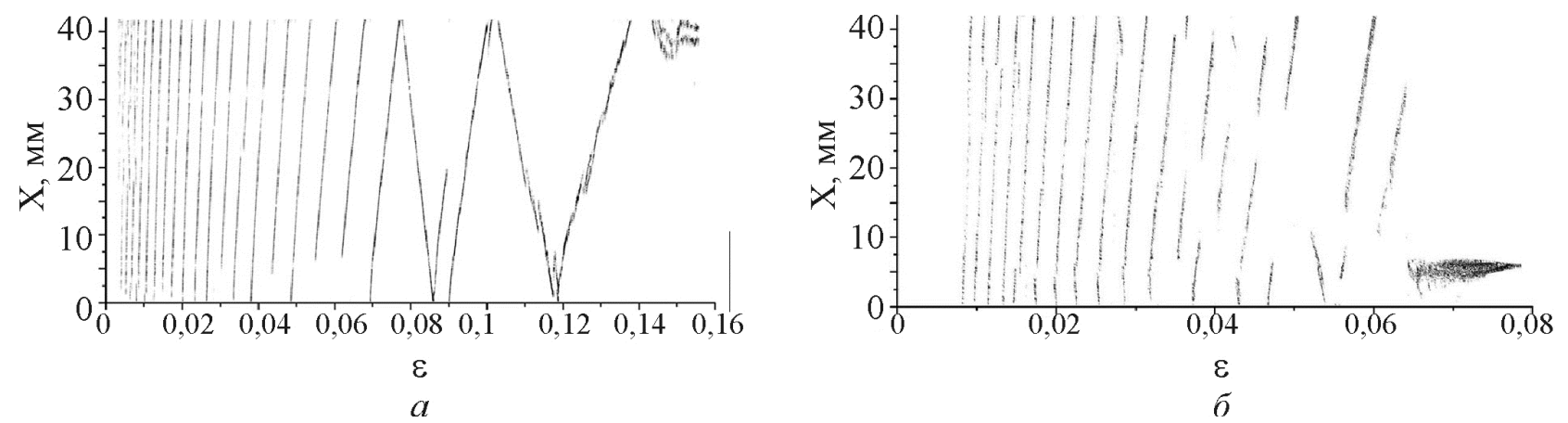

Рис. 4. - Кинетические диаграммы полос локализованной деформации в сплаве Д1 в исходном состоянии ( $a$ ) и после электролитического насыщения (б)

\section{4. Заключение}

Водородное охрупчивание сплавов является серьезной практической проблемой [2-5], от успешного решения которой зависит безопасность работы конструкций. В настоящей работе показано влияние насыщения водородом алюминиевого сплава марки Д1 электролитическим методом на локализацию пластического течения. Насыщение водородом образцов в течение 100 ч в значительной степени влияет на пластичность сплава по сравнению с исходным состоянием. При этом остается неизменным число присутствующих стадий пластического течения, а именно: линейная, параболическая и стадия предразрушения, однако сокращается их продолжительность.

Водородное охрупчивание дуралюмина происходит по электрохимическому механизму путем периодического разрушения пассивной пленки за счет локальной пластической деформации у границ зерен и преимущественного локального растворения в этих местах [2-6]. Для объяснения водородных эффектов развито много микроскопических моделей, например [17], базирующихся на теории дислокаций. Однако такие подходы представляются недостаточными, поскольку не учитывают того обстоятельства, что пластическая деформация твердых тел развивается локализовано на всем протяжении процесса течения [10, 11, 18-20]. Особенно эффектно этот процесс проявляется на макромасштабном уровне, когда формирующиеся локализационные паттерны оказываются однозначно связанными с законами деформационного упрочнения $\theta(\varepsilon)$, действующими на соответствующей стадии процесса. В этом случае паттерны принимают форму автоволн разных типов $[10,11]$. В настоящей работе показано, что водород усиливает локализацию пластической деформации и меняет количественные параметры локализации пластической деформации: скорость фронтов локализации пластической деформации и расстояние между ними.

\section{Благодарность}

Работа выполнена в рамках Программы фундаментальных исследований Государственной академии наук в 2015-2020 гг. и Программы «Научный фонд им. Д.И. Менделеева Томского государственного университета» в 2016 г и гранта РФФИ № 16-08-00385-а.

\section{Литература}

1. Семенова И. В., Флорианович Г. М., Хорошилов А. В. Коррозия и защита от коррозии. - М. : Физматлит, 2002. - 335 с.

2. Lunarska E., Chernyaeva O. Effect of precipitates on hydrogen transport and hydrogen embrittlement of aluminum alloys // Materials Science. - 2004. - Vol. 40, № 3. - P. 399-407. DOI: $10.1007 / \mathrm{s} 11003-005-0049-2$. 
3. Kannan M., Raja V. S Hydrogen embrittlement susceptibility of over aged 7010 Al-alloy // Journal of Materials Science. - 2006. - Vol. 41. - P. 5495-5499. - DOI: 10.1007/s10853-006-0287-1.

4. Kim S. J., Han M. S., Jang S. K. Electrochemical characteristics of Al-Mg alloy in seawater for leisure ship: Stress corrosion cracking and hydrogen embrittlement // Korean Journal of Chemical Engineering. - 2009. - Vol. 26, № 1. - P. 250-257. - DOI: 10.1007/s11814-009-0042-9.

5. Kumar S., Namboodhiri T. K. G. Precipitation hardening and hydrogen embrittlement of aluminum alloy AA7020 // Bulletin of Materials Science. - 2011. - Vol. 34, no. 2. - P. 311-321. DOI: $10.1007 / \mathrm{s} 12034-011-0066-8$.

6. Electrochemical evaluation of the in-service degradation of an aircraft aluminum alloy / H. M. Nykyforchyn, O. P. Ostash, O. T. Tsyrul'nyk, I. M. Andreiko, Yu. V. Holovatyuk // Materials Science. - 2008. - Vol. 44, no. 2. - P. 254-259. - DOI: 10.1007/s11003-008-9067-1.

7. Plekhov O.A., Naimark O., Saintier N. Experimental study of energy accumulation and dissipation in iron in an elastic-plastic transition // Technical Physics. - 2007. - Vol. 52, no. 9. P. 1236-1238. - DOI: 10.1134/S106378420709023X.

8. Третьякова Т. В., Вильдеман В. Э. Исследование развития трещин при сложных режимах нагружения методом корреляции цифровых изображений // Заводская лаборатория Диагностика материалов. - 2012. - Т. 78, № 6. - С. 54-58.

9. Шибков А. А., Золотов А. Е., Желтов М. А. Механизмы зарождения полос макролокализованной деформации // Известия РАН. Серия физическая. - 2012. - Т. 76, № 1. - С. 85-95.

10. Danilov V. I., Bochkaryova A. V., Zuev L. B.. Macrolocalization of deformation in material having unstable plastic flow behavior // The Physics of Metals and Metallography - 2009. Vol. 107, iss. 6. - P. 616-623. - DOI: 10.1134/S0031918X0906012X.

11. Zuev L. B., Gorbatenko V. V., Pavlichev K. V. Elaboration of speckle photography techniques for plastic flow analyses // Measurement Science and Technology. - 2010. - Vol. 21, no. 5. - P. 1-5. - DOI: 10.1088/0957-0233/21/5/054014.

12. Lattice defects dominating hydrogen-related failure of metals / K. Takai, H. Shoda, H. Suzuki, M. Nagumo // Acta Materialia. - 2008. - Vol. 56, iss. 18. - P. 5158-5167. DOI: 10.1016/j.actamat.2008.06.031.

13. Hydrogen Solubility and Diffusion in Austenitic Stainless Steels Studied with Thermal Desorption Spectroscopy / Y. Yagodzinskyy, O. Todoshchenko, S. Papula, H. Hänninen // Steel Research International. - 2011. - Vol. 82, iss. 1. - P. 20-25. - DOI: 10.1002/srin.201000227.

14. Tensile plastic strain localization in single crystals of austenite steel electrolytically saturated with hydrogen / S. A. Barannikova, M. V. Nadezhkin, V. A. Mel'nichuk, L. B. Zuev // Technical Physics Letters. - 2011. - Vol. 37, no. 9. - P. 793-796. - DOI: 10.1134/S1063785011090057.

15. Effect of hydrogen on the localization of plastic deformation under tensile of low-carbon steel / S. A. Barannikova, M. V. Nadezhkin, A. G. Lunev, V. V. Gorbatenko, G. V. Shlyakhova, L. B. Zuev // Metallofizica i Noveyshie Tekhnologii. - 2014. - Vol. 36, iss. 2. - P. 229-245.

16. Regularities in localization of plastic flow upon electrolytic hydrogenation of an iron bccalloy / S. A. Barannikova, M. V. Nadezhkin, A. G. Lunev, V. V. Gorbatenko, L. B. Zuev // Technical Physics Letters. - 2014. - Vol. 40, iss. 3. - P. 211-214. - DOI: 10.1134/S1063785014030043.

17. Birnbaum H. K., Sofronis P. Hydrogen-enhanced localized plasticity - a mechanism for hydrogen-related fracture // Material Science and Engineering: A. - 1994. - Vol. 176, iss. 1-2. P. 191-202. - DOI: 10.1016/0921-5093(94)90975-X.

18. McDonald R. J., Efstathiou C., Curath P. The wave-like plastic deformation of single crystals copper // Journal of Engineering Materials and Technology. - 2009. - Vol. 131, no. 4. P. 692-703. - DOI: 10.1115/1.3120410.

19. Asharia A., Beaudoin A., Miller R. New perspectives in plasticity theory: dislocation nucleation, waves and partial continuity of plastic strain rate // Mathematics and Mechanics of Solids. - 2008. - Vol. 13, no. 3-4. - P. 292-315. - DOI: 10.1177/1081286507086903. 
20. Fressengeas C., Beaudoin A., Entemeyer D. Dislocation transport and intermittency in the plasticity of crystalline solids // Physical Review B. - 2009. - Vol. 79. - P. 014108-1-014108-9. DOI: 0.1103/B.79.014108. 